\title{
PBW Basis of Non-standard Quantum Groups $X_{q}\left(A_{n}\right)$
}

\author{
Cheng Cheng*, Shilin Yang \\ College of Applied Sciences, Beijing University of Technology, Beijing 100124, P. R. China. \\ * Corresponding author. Tel.: 18810925969; email: tanchengxiangdai@yeah.net \\ Manuscript submitted April 19, 2017; accepted May 16, 2017. \\ doi: 10.17706/ijapm.2017.7.3.191-199
}

\begin{abstract}
A kind of non-standard quantum group $X_{q}\left(A_{n}\right)$ is studied in the paper. Root vectors of $X_{q}\left(A_{n}\right)$ and their commutation relation are described. Then we establish the PBW basis of $X_{q}\left(A_{n}\right)$.
\end{abstract}

Key words: Non-standard quantum group, PBW basis, root vector.

\section{Introduction}

Throughout the paper, we always assume that the base field is the complex number field $\mathbb{C}$ and

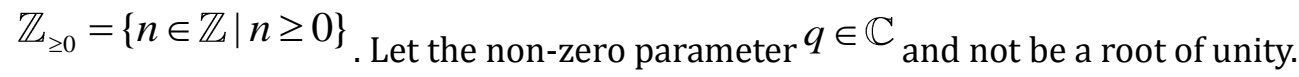

Quantum groups always are the hot research topic in mathematics and physics after they were introduced by Drinfeld and Jimbo in 1980s. Ge et al. [1] constructed a new quantum group by solving exotic solution of quantum Yang-Baxter equation, which is also called the non-standard quantum group. Jing et al. [2] derived a new quantum group $X_{q}(2)$ and described all finite dimensional irreducible representations of $X_{q}(2)$. Aghamohammadi et al. [3] obtained a non-standard quantum group $X_{q}\left(A_{n-1}\right)$ corresponding to type $A_{n-1}$. It is noted that $X_{q}\left(A_{1}\right)$ is just quantum algebra $X_{q}(2)$. In 1994, Aghamohammadi et al. [4] constructed the non-standard quantum group $X_{q}\left(B_{n}\right)$ corresponding to the series $B_{n}$. Cheng and Yang [5] construct a weak Hopf algebra $w X_{q}\left(A_{1}\right)$ corresponding to non-standard quantum group $X_{q}\left(A_{1}\right)$, and describe the PBW basis of $w X_{q}\left(A_{1}\right)$.

In this paper, we describe the PBW basis of a particular class of $X_{q}\left(A_{n}\right)$. The paper is arranged as follows. In Section 2, we rewrite definition of the Hopf algebra $X_{q}\left(A_{n}\right)$ referred to the quantum algebra $X_{q}\left(A_{n}\right)$ [3]. In Section 3, we establish the root vectors and investigate commutation relations of $X_{q}\left(A_{n}\right)$ in the case of $q_{i}=q(0 \leq i \leq n)$ and $q_{n+1}=-q^{-1}$. In Section 4 , we construct the PBW basis of $X_{q}\left(A_{n}\right)$ as described in Section 3.

\section{Preliminaries}

In first, we have quantum group $X_{q}\left(A_{n}\right)$ by replacing some generators in [3].

Definition 2. 1. $X_{q}\left(A_{n}\right)$ is an associative algebra over the field $\mathbb{C}$ with 1 generated by 
$K_{1}^{ \pm 1}, K_{2}^{ \pm 1}, \ldots, K_{n}^{ \pm 1}$,

$K_{n+1}^{ \pm 1}, E_{1}, \ldots, E_{n}, F_{1}, \ldots, F_{n}$ with the following relations:

(R1) $K_{i} K_{i}^{-1}=K_{i}^{-1} K_{i}=1, K_{i} K_{j}=K_{j} K_{i}, \quad$ (R2) $K_{i} E_{j}=E_{j} K_{i}, K_{i} F_{j}=F_{j} K_{i}, i \neq j, j+1$,

(R3) $K_{i} E_{i}=q_{i}^{-1} E_{i} K_{i}, K_{i} F_{i}=q_{i} F_{i} K_{i}$,

(R4) $K_{i+1} E_{i}=q_{i+1} E_{i} K_{i+1}, K_{i+1} F_{i}=q_{i+1}^{-1} F_{i} K_{i+1}$,

(R5) $E_{i} F_{j}-F_{j} E_{i}=\delta_{i, j} \frac{K_{i+1} K_{i}^{-1}-K_{i} K_{i+1}^{-1}}{q-q^{-1}}$,

(R6) $\left(q_{i}-q_{i+1}\right) E_{i}^{2}=\left(q_{i}-q_{i+1}\right) F_{i}^{2}=0$,

(R7) $q_{i} E_{i}^{2} E_{i \pm 1}-\left(1+q_{i} q_{i+1}\right) E_{i} E_{i \pm 1} E_{i}+q_{i+1} E_{i \pm 1} E_{i}^{2}=0$,

(R8) $q_{i} F_{i}^{2} F_{i \pm 1}-\left(1+q_{i} q_{i+1}\right) F_{i} F_{i \pm 1} F_{i}+q_{i+1} F_{i \pm 1} F_{i}^{2}=0$,

where $q_{i}=\mathrm{q}$ or $-q^{-1}$. If all $q_{i}=q(0 \leq i \leq n+1)$, then $X_{q}\left(A_{n}\right)$ is similar to $U_{q}\left(s l_{n+1}\right)$. If $q_{i} \neq q_{i+1}$ for some $1 \leq i \leq n$, then $E_{i}^{2}=F_{i}^{2}=0$. The relations is different from $U_{q}\left(s l_{n+1}\right)$.

Proposition 2. 2. Keeping notations as above. Then $X_{q}\left(A_{n}\right)$ is a Hopf algebra with comultiplication $\Delta$, counit $\varepsilon$ and antipode $S$, which are defined as following

$$
\begin{aligned}
& \Delta: X_{q}\left(A_{n}\right) \rightarrow X_{q}\left(A_{n}\right) \otimes X_{q}\left(A_{n}\right), \Delta\left(K_{i}\right)=K_{i} \otimes K_{i}, \Delta\left(K_{i}^{-1}\right)=K_{i}^{-1} \otimes K_{i}^{-1}, \\
& \Delta\left(E_{i}\right)=K_{i} K_{i+1}^{-1} \otimes E_{i}+E_{i} \otimes 1, i \neq n, \Delta\left(F_{i}\right)=1 \otimes F_{i}+F_{i} \otimes\left(K_{i}^{-1} K_{i+1}\right), i \neq n, \\
& \mathrm{~S}: X_{q}\left(A_{n}\right) \rightarrow X_{q}\left(A_{n}\right), S\left(K_{i}\right)=K_{i}^{-1}, S\left(K_{i}^{-1}\right)=K_{i}, S\left(E_{i}\right)=-K_{i+1} K_{i}^{-1} E_{i}, i \neq n, \\
& S\left(F_{i}\right)=-F_{i} K_{i} K_{i+1}^{-1}, i \neq n, S\left(F_{n}\right)=-F_{n} K_{n}, \\
& \varepsilon: X_{q}\left(A_{n}\right) \rightarrow \mathbb{C}, \varepsilon\left(K_{i}\right)=\varepsilon\left(K_{i}^{-1}\right)=1, \varepsilon\left(E_{i}\right)=\varepsilon\left(F_{i}\right)=0 .
\end{aligned}
$$

Proof. The proof is more or less the same as that in [6, Proposition VII.1.1].

\section{Commutation relations between root vectors}

In first, we recall the definition of operator ad. Assume that $H$ is a Hopf algebra and $x \in H$, we can define the adjoint operator $\operatorname{ad}_{x}: H \rightarrow H$, associated to $x$ as $\operatorname{ad}_{x}(y)=\sum x_{(1)} y S\left(x_{(2)}\right)$ for all $y \in H$, where $\Delta(x)=\sum_{(x)} x_{(1)} \otimes x_{(2)}$.

Let $V=\mathbb{R}^{n+1}$ be an $n+1$-dimensional Euclidean space with $\varepsilon_{1}, \varepsilon_{2}, \ldots, \varepsilon_{n}, \varepsilon_{n+1}$ as an orthogonal basis of $V$. Each positive root $\alpha$ can be written as $\alpha=\varepsilon_{i}-\varepsilon_{j}=\alpha_{i}+\alpha_{i+1}+\ldots+\alpha_{j-1}, 1 \leq i, j \leq n+1$. We define root vectors by $E_{i, j}:=\operatorname{ad}_{E_{i}}\left(E_{i+1, j}\right), 1 \leq i, j \leq n$, corresponding to the root $\varepsilon_{i}-\varepsilon_{j}$, specially, $E_{i, i+1}:=E_{i}$. Define the order of all positive roots by

$$
\alpha_{1}>\alpha_{1}+\alpha_{2}>\ldots>\alpha_{1}+\alpha_{2}+\ldots+\alpha_{n}>\alpha_{2}>\alpha_{2}+\alpha_{3}>\ldots>\alpha_{2}+\ldots+\alpha_{n}>\ldots>\alpha_{n-1}>\alpha_{n-1}+\alpha_{n}>\alpha_{n} .
$$

The algebra $X_{q}\left(A_{n}\right)$ is graded via $\operatorname{deg} E_{i}=\alpha_{i}, \operatorname{deg} F_{i}=-\alpha_{i}, \operatorname{deg} K_{i}=\operatorname{deg} K_{i}^{-1}=0$.

Set $\xi($ resp. $\eta)$ be root vector corresponding to positive root $\beta$ (resp. $\gamma$ ). We also can say that $\xi$ (resp. $\eta \in X_{q}\left(A_{n}\right)$ is of degree $\beta($ resp. $\gamma)$. We denote $K_{i} K_{i+1}^{-1} \xi=t_{i \beta} \xi$. Then we have the following formula.

Proposition 3. 1 We have $a d_{E_{i}}(\xi \eta)=a d_{E_{i}}(\xi) \eta+t_{i \beta} \xi a d_{E_{i}}(\eta)$, where 


$$
t_{i, \beta}= \begin{cases}1, & \text { if } \alpha_{i-1}, \alpha_{i}, \alpha_{i+1} \in \beta, \text { or } \alpha_{i-1}, \alpha_{i}, \alpha_{i+1} \notin \beta ; \\ q_{i+1}, & \text { if } \alpha_{i+1} \in \beta, \text { and } \alpha_{i-1}, \alpha_{i} \notin \beta ; \\ q_{i}^{-1} q_{i+1}^{-1} q_{i+1}=q_{i}^{-1}, & \text { if } \alpha_{i}, \alpha_{i+1} \in \beta, \text { and } \alpha_{i-1} \notin \beta ; \\ q_{i}^{-1} q_{i+1}^{-1}, & \text { if } \beta=\alpha_{i} ; \\ q_{i}, & \text { if } \alpha_{i-1} \in \beta, \text { and } \alpha_{i} \alpha_{i+1} \notin \beta ; \\ q_{i+1}^{-1}, & \text { if } \alpha_{i-1}, \alpha_{i} \in \beta, \text { and } \alpha_{i+1} \notin \beta .\end{cases}
$$

Proof. Note that $t_{i(\beta+\gamma)}=t_{i \beta} t_{i \gamma}$, we have $\operatorname{ad}_{E_{i}}(\xi \eta)=E_{i} \xi \eta+t_{i(\beta+\gamma)}(\xi \eta) E_{i}, \quad \operatorname{ad}_{E_{i}}(\xi) \eta=E_{i} \xi \eta-t_{i(\beta)} \xi E_{i} \eta$, $\xi a d_{E_{i}}(\eta)=\xi E_{i} \eta-t_{i(\gamma)} \xi a d_{E_{i}}(\eta), \quad a d_{E_{i}}(\xi \eta)=a d_{E_{i}}(\xi) \eta+t_{i \beta} \xi a d_{E_{i}}(\eta)$.

Next we investigate commutation relations of $X_{q}\left(A_{n}\right)$. We consider $X_{q}\left(A_{n}\right)$ in the case of $q_{i}=q(0 \leq i \leq n), q_{n+1}=-q^{-1}$ in the sequel. We have (R6') $E_{n}^{2}=F_{n}^{2}=0$,

$\left(\mathrm{R}^{\prime}\right) E_{i}^{2} E_{i \pm 1}-\left(q^{-1}+q\right) E_{i} E_{i \pm 1} E_{i}+E_{i \pm 1} E_{i}^{2}=0(i \neq n),\left(\mathrm{R} 8^{\prime}\right) F_{i}^{2} F_{i \pm 1}-\left(q^{-1}+q\right) F_{i} F_{i \pm 1} F_{i}+F_{i \pm 1} F_{i}^{2}=0(i \neq n)$.

Now, we describe the commutation relations between $E_{k}$ and $E_{\varepsilon_{i}-\varepsilon_{j}}$.

Lemma 3. 2. For all $0 \leq i, j \leq n+1$,

(1) If $k=i-1$, then $E_{k} E_{i, j+1}-q_{i} E_{i, j+1} E_{k}=E_{i-1, j+1}$.

(2) If $k=j+1 \neq n$, then $E_{i, j+1} E_{j+1}-q_{j+1} E_{j+1} E_{i, j+1}=E_{i, j+2}$.

(3) ${ }_{\text {If }} k \leq i-2$ or $k \geq j+2$, then $E_{k} E_{i, j+1}=E_{i, j+1} E_{k}$.

(4) ${ }_{\text {If }} k=i$, then $E_{k} E_{i, j+1}=q_{i}^{-1} E_{i, j+1} E_{k}$.

(5) If $i+1 \leq k \leq j-1$, then $E_{k} E_{i, j+1}=E_{i, j+1} E_{k}$.

(6) If $k=j$, then $E_{j} E_{j-1, j+1}=q_{j+1} E_{j-1, j+1} E_{j}$ (if $E_{j}^{2} \neq 0$ ), $E_{j} E_{j-1, j+1}=q_{j}^{-1} E_{j-1, j+1} E_{j}$ (if $E_{j}^{2}=0$ ).

Proof. (1) If $k=i-1$, by definition we have $a d_{E_{k}}\left(E_{i, j+1}\right)=E_{k} E_{i, j+1}-q_{i} E_{i, j+1} E_{k}=E_{\varepsilon_{i-1}-\varepsilon_{j+1}}=E_{i-1, j+1}$.

(2) If $k=j+1 \neq n$, then we have $E_{j+1} E_{i, j+1}=a d_{E_{i}} a d_{E_{i}+1} \ldots a d_{E_{j-1}}\left(E_{j}\right)=a d_{E_{i}} a d_{E_{i}+1} \ldots a d_{E_{j-1}}\left(E_{j+1} E_{j}\right)$, $E_{i, j+1} E_{j+1}=a d_{E_{i}} a d_{E_{i}+1} \ldots a d_{E_{j-1}}\left(E_{j} E_{j+1}\right)$, and $a d_{E_{j}}\left(E_{j+1}\right)=-q_{j+1} E_{j+1} E_{j}+E_{j} E_{j+1}$.

So $E_{i, j+1} E_{j+1}-q_{j+1} E_{j+1} E_{i, j+1}=E_{i, j+2}$.

(3) $k \leq i-2$ or $k \geq j+2$, it is easy to see that $E_{k} E_{i, j+1}=E_{i, j+1} E_{k}$.

(4) $k=i$, if $E_{i}^{2} \neq 0$, as the proof in [7], we have $a d_{E_{i}}\left(E_{i, j+1}\right)=a d_{E_{i}} a d_{E_{i}} a d_{E_{i+1}}\left(E_{i+2, j+1}\right)=\left(q_{i}^{-1}+q_{i+1}\right) a d_{E_{i}} a d_{E_{i+1}} a d_{E_{i}}-q_{i}^{-1} q_{i+1} a d_{E_{i}}^{2}\left(E_{i+2, j+1}\right)=0$. If $E_{i}^{2}=0, q_{i+1}=-q_{i}^{-1}$, $a d_{E_{i}}\left(E_{i, j+1}\right)=E_{i} E_{i} E_{i+1, j+1}-q_{i+1} E_{i} E_{i+1, j+1} E_{i}+\left(K_{i} K_{i+1}^{-1} E_{i} E_{i+1, j+1} S\left(E_{i}\right)-q_{i+1} K_{i} K_{i+1}^{-1} E_{i+1, j+1} E_{i} S\left(E_{i}\right)\right)$ $=-q_{i+1} E_{i} E_{i+1, j+1} E_{i}-K_{i} K_{i+1}^{-1} E_{i} E_{i+1, j+1} K_{i+1} K_{i}^{-1}=\left(-q_{i+1}-q_{i}^{-1}\right) E_{i} E_{i+1, j+1} E_{i}=0$. Also $\operatorname{ad}_{E_{i}}\left(E_{i, j+1}\right)=E_{k} E_{i, j+1}-q_{i}^{-1} E_{i, j+1} E_{k}$, So $E_{k} E_{i, j+1}=q_{i}^{-1} E_{i, j+1} E_{k}$. 
(5) If $i+1 \leq k \leq j-1$, , it is similar to the proof in [7]. We have $a d_{E_{k}}\left(E_{i, j+1}\right) E_{k} E_{i, j+1}-E_{i, j+1} E_{k} a d_{E_{i}} \ldots a d_{E_{k-2}} a d_{E_{k}} a d_{E_{k-1}} a d_{E_{k}}\left(E_{k+1, j+1}\right)=0$.

(6) If $k=j$, then $E_{j} E_{i, j+1}=a d_{E_{i}} a d_{E_{i+1}} \ldots a d_{E_{j-2}}\left(E_{j} E_{j-1, j+1}\right)$,

$E_{i, j+1} E_{j}=a d_{E_{i}} a d_{E_{i+1}} \ldots a d_{E_{j-2}}\left(E_{j-1, j+1} E_{j}\right), E_{j-1, j+1}=a d_{j-1}\left(E_{j}\right)=-q_{j} E_{j} E_{j-1}+E_{j-1} E_{j}$,

$E_{j} E_{j-1, j+1}=-q_{j} E_{j}^{2} E_{j-1}+E_{j} E_{j-1} E_{j}, E_{j-1, j+1} E_{j}=-q_{j} E_{j} E_{j-1} E_{j}+E_{j-1} E_{j}^{2}$. So we have

$E_{j} E_{j-1, j+1}-q_{j+1} E_{j-1, j+1} E_{j}=0\left(E_{j}^{2} \neq 0\right), q_{j} E_{j} E_{j-1, j+1}-E_{j-1, j+1} E_{j}=0\left(E_{j}^{2}=0\right)$.

The proof is finished.

Remark 3. 3. It is noted that Lemma $3.2(1)(2)(3)(4)(6)$ also hold when $X_{q}\left(A_{n}\right)$ is in the case of $q_{i} \neq q_{i+1}(i \neq n)$.

Let $\alpha=\alpha_{i}+\ldots+\alpha_{p}, \beta=\alpha_{j}+\ldots+\alpha_{k}$. Next we consider the relations between $E_{\alpha}$ and $E_{\beta}$.

Lemma 3. 4. (1) If $j \geq p+2$, then $E_{\alpha} E_{\beta}=E_{\beta} E_{\alpha}$.

(2) If $j=p+1$, then $E_{\alpha} E_{\beta}-q_{p+1} E_{\beta} E_{\alpha}=E_{\alpha+\beta}$.

(3) If $j \leq p$, then $E_{\alpha} E_{\beta}-E_{\beta} E_{\alpha}=\left(q_{j}^{-1}-q_{j}\right) E_{\alpha+\beta-\gamma} E_{\gamma}$.

Proof. (1) $j \geq p+2$, it is easy to see $E_{\alpha} E_{\beta}=E_{\beta} E_{\alpha}$.

(2) If $j=p+1$, we have $E_{\alpha}=a d_{E_{i}} a d_{E_{i+1}} \ldots a d_{E_{p-2}}\left(-q_{p} E_{p} E_{p-1}+E_{p-1} E_{p}\right)=E_{i, p} E_{p}-q_{p} E_{p} E_{i, p}$.

$$
\begin{aligned}
& E_{\alpha} E_{\beta}=E_{i, p} E_{p} E_{\beta}-q_{p} E_{p} E_{i, p} E_{\beta}=E_{i, p} E_{p} E_{\beta}-q_{p} E_{p} E_{\beta} E_{i, p}, \\
& E_{\beta} E_{\alpha}=E_{\beta} E_{i, p} E_{p}-q_{p} E_{\beta} E_{p} E_{i, p}=E_{i, p} E_{\beta} E_{p}-q_{p} E_{\beta} E_{p} E_{i, p},
\end{aligned}
$$

Also $E_{p} E_{\beta}-q_{p+1} E_{\beta} E_{p}=E_{p+\beta}$. Then $E_{\alpha} E_{\beta}-q_{p+1} E_{\beta} E_{\alpha}=E_{\alpha+\beta}$.

(3) If $j \leq p$, we assume that $\gamma=\alpha_{j}+\alpha_{j+1}+\ldots+\alpha_{p}, \alpha=\alpha_{i}+\ldots+\alpha_{j-1}+\gamma, \beta=\gamma+\alpha_{p+1}+\ldots+\alpha_{k}$.

So we have $E_{\alpha} E_{\beta}=a d_{E_{i}} \ldots a d_{E_{j-2}}\left(E_{\alpha_{j-1}+\gamma} E_{\beta}\right), E_{\beta} E_{\alpha}=a d_{E_{i}} \ldots a d_{E_{j-2}}\left(E_{\beta} E_{\alpha_{j-1}+\gamma}\right)$

$$
E_{\alpha_{j-1}+\gamma} E_{\beta}=a d_{E_{j-1}}\left(E_{\gamma} E_{\beta}\right)-q_{j} E_{\gamma} E_{\alpha_{j-1}+\beta}, E_{\beta} E_{\alpha_{j-1}+\gamma}=q_{j}^{-1}\left(a d_{E_{j-1}}\left(E_{\beta} E_{\gamma}\right)-E_{\alpha_{j-1}+\beta} E_{\gamma}\right) .
$$

We also know that $E_{j}, E_{j+1}, \ldots, E_{p}$ are commutative with $E_{\alpha_{j-1}+\beta}$, then $E_{\alpha_{j-1}+\beta} E_{\gamma}=E_{\gamma} E_{\alpha_{j-1}+\beta}$. And set $\gamma=\alpha_{j}+\gamma^{\prime}$, also we have $E_{\beta} E_{\gamma^{\prime}}=E_{\gamma^{\prime}} E_{\beta}$. Then $E_{\gamma} E_{\beta}=a d_{E_{j}}\left(E_{\gamma^{\prime}} E_{\beta}\right)$,

$$
E_{\beta} E_{\gamma}=E_{\beta} a d_{E_{j}}\left(E_{\gamma^{\prime}}\right)=q_{j} a d_{E_{j}}\left(E_{\beta} E_{\gamma^{\prime}}\right), E_{\gamma} E_{\beta}=q_{j}^{-1} E_{\beta} E_{\gamma} .
$$

Then we get $E_{\alpha_{j-1}+\gamma} E_{\beta}-E_{\beta} E_{\alpha_{j-1}+\gamma}=-q_{j} E_{\gamma} E_{\alpha_{j-1}+\gamma}+q_{j}^{-1} E_{\alpha_{j-1}+\gamma} E_{\gamma}=\left(q_{j}^{-1}-q_{j}\right) E_{\alpha_{j-1}+\gamma} E_{\gamma}$.

Hence,

$$
E_{\alpha} E_{\beta}-E_{\beta} E_{\alpha}=a d_{E_{i}} \ldots a d_{E_{j-2}}\left(\left(q_{j}^{-1}-q_{j}\right) E_{\alpha_{j-1}+\gamma} E_{\gamma}\right)=\left(q_{j}^{-1}-q_{j}\right) E_{\alpha+\beta-\gamma} E_{\gamma} .
$$

The proof is finished. 
Lemma 3. 5. $\Delta\left(E_{i, j+1}\right)=K_{i} K_{j+1}^{-1} \otimes E_{i, j+1}+E_{i, j+1} \otimes 1+\sum_{k=i}^{j-1}\left(1-q_{k+1}^{2}\right) E_{i, k+1} K_{k+1} K_{j+1}^{-1} \otimes E_{k+1, j+1}$. Proof. We use induction on $E_{i, j+1}$ to prove the formula. Assume that

$$
\Delta\left(E_{i+1, j+1}\right)=K_{i+1} K_{j+1}^{-1} \otimes E_{i+1, j+1}+E_{i+1, j+1} \otimes 1+\sum_{k=i+1}^{j-1}\left(1-q_{k+1}^{2}\right) E_{i+1, k+1} K_{k+1} K_{j+1}^{-1} \otimes E_{k+1, j+1}
$$

holds,

then

$$
\begin{aligned}
& \Delta\left(E_{i, j+1}\right)=\Delta\left(E_{i} E_{i+1, j+1}-q_{i+1} E_{i+1, j+1} E_{i}\right)=\left(K_{i} K_{i+1}^{-1} \otimes E_{i}+E_{i} \otimes 1\right) \Delta\left(E_{i+1, j+1}\right)-q_{i+1} \Delta\left(E_{i+1, j+1}\right)\left(K_{i} K_{i+1}^{-1} \otimes E_{i}+E_{i} \otimes 1\right) \\
& =K_{i} K_{i+1}^{-1} \otimes E_{i} E_{i+1, j+1}+K_{i} K_{i+1}^{-1} E_{i+1, j+1} \otimes E_{i}+\sum_{k=i+1}^{j-1}\left(1-q_{k+1}^{2}\right) K_{i} K_{i+1}^{-1} E_{i+1, k+1} K_{k+1} K_{j+1}^{-1} \otimes E_{i} E_{k+1, j+1} \\
& +E_{i} K_{i+1} K_{j+1}^{-1} \otimes E_{i+1, j+1}+E_{i} E_{i+1, j+1} \otimes 1+\sum_{k=i+1}^{j-1}\left(1-q_{k+1}^{2}\right) E_{i} E_{i+1, k+1} K_{k+1} K_{j+1}^{-1} \otimes E_{k+1, j+1} \\
& -q_{i+1}\left(K_{i} K_{j+1}^{-1} \otimes E_{i+1, j+1} E_{i}+K_{i+1} K_{j+1}^{-1} E_{i} \otimes E_{i+1, j+1}+E_{i+1, j+1} K_{i} K_{j+1}^{-1} \otimes E_{i}+E_{i+1, j+1} E_{i} \otimes 1\right. \\
& +\sum_{k=i+1}^{j-1}\left(1-q_{k+1}^{2}\right) E_{i+1, k+1} K_{k+1} K_{j+1}^{-1} K_{i} K_{i+1}^{-1} \otimes E_{k+1, j+1} E_{i}+\sum_{k=i+1}^{j-1}\left(1-q_{k+1}^{2}\right) E_{i+1, k+1} K_{k+1} K_{j+1}^{-1} E_{i} \otimes E_{k+1, j+1} \\
& =K_{i} K_{j+1}^{-1} \otimes E_{i, j+1}+E_{i, j+1} \otimes 1+E_{i} K_{i+1} K_{j+1}^{-1} \otimes E_{i+1, j+1}-q_{i+1}^{2} E_{i} K_{i+1} K_{j+1}^{-1} \otimes E_{i+1, j+1}+\sum_{k=i+1}^{j-1}\left(1-q_{k+1}^{2}\right) E_{i, k+1} K_{k+1} K_{j+1}^{-1} \otimes E_{k+1, j+1} \\
& =K_{i} K_{j+1}^{-1} \otimes E_{i, j+1}+E_{i, j+1} \otimes 1+\sum_{k=i}^{j-1}\left(1-q_{k+1}^{2}\right) E_{i, k+1} K_{k+1} K_{j+1}^{-1} \otimes E_{k+1, j+1} \cdot
\end{aligned}
$$

The proof is finished.

\section{PBW Basis of Nonstandard Quantum Group $X_{q}\left(A_{n}\right)$}

To describe the PBW basis of $X_{q}\left(A_{n}\right)$, we need to prove the following equality

Lemma 4.1. $q_{i} E_{i}^{2} E_{i+1, n+1}-\left(1+q_{i} q_{i+1}\right) E_{i} E_{i+1, n+1} E_{i}+q_{i+1} E_{i+1, n+1} E_{i}^{2}=0(0 \leq i \leq n-1)$.

Proof. $q_{i} E_{i}^{2} E_{i+1, n+1}-\left(1+q_{i} q_{i+1}\right) E_{i} E_{i+1, n+1} E_{i}+q_{i+1} E_{i+1, n+1} E_{i}^{2}$

$=q_{i} E_{i}^{2}\left(E_{i+1} E_{i+2, n+1}-q_{i+2} E_{i+2, n+1} E_{i+1}\right)-\left(1+q_{i} q_{i+1}\right) E_{i}\left(E_{i+1} E_{i+2, n+1}-q_{i+2} E_{i+2, n+1} E_{i+1}\right) E_{i}+q_{i+1}\left(E_{i+1} E_{i+2, n+1}-q_{i+2} E_{i+2, n+1} E_{i+1}\right) E_{i}^{2}$

$=-q_{i+2} E_{i+2, n+1}\left(q_{i} E_{i}^{2} E_{i+1}-\left(1+q_{i+1} q_{i}\right) E_{i} E_{i+1} E_{i}+q_{i+1} E_{i+1} E_{i}^{2}\right)+\left(q_{i} E_{i}^{2} E_{i+1}-\left(1+q_{i+1} q_{i}\right) E_{i} E_{i+1} E_{i}+q_{i+1} E_{i+1} E_{i}^{2}\right) E_{i+2, n+1}$

$=0$.

The proof is finished.

Lemma 4.2. Keeping notations as above, we have $E_{i, n+1}^{2}=0(1 \leq i \leq n)$, where $E_{i, n+1}$ is a root vector which contain $\alpha_{n}$.

Proof. We verify the equality by induction on the root.

$E_{\alpha_{n-1}+\alpha_{n}}^{2}=E_{n-1, n+1}^{2}=\left(E_{n-1} E_{n}-q_{n} E_{n} E_{n-1}\right)=q_{n}^{2}\left(E_{n} E_{n-1}\right)^{2}+\left(E_{n-1} E_{n}\right)^{2}-q_{n} E_{n} E_{n-1^{2}} E_{n}$

$=q_{n}^{2}\left(E_{n} E_{n-1}\right)^{2}+\left(E_{n-1} E_{n}\right)^{2}-q_{n} E_{n}\left(\left(q_{n-1}^{-1}+q_{n}\right) E_{n-1} E_{n} E_{n-1}-q_{n-1}^{-1} q_{n} E_{n} E_{n-1}^{2}\right)$

$=q_{n}^{2}\left(E_{n} E_{n-1}\right)^{2}+\left(E_{n-1} E_{n}\right)^{2}-q_{n}\left(q_{n-1}^{-1}+q_{n}\right)\left(E_{n} E_{n-1}\right)^{2}=\left(E_{n-1} E_{n}\right)^{2}-q_{n} q_{n-1}^{-1}\left(E_{n} E_{n-1}\right)^{2}$.

Also $\left(q_{n-1} E_{n-1}^{2} E_{n}-\left(1+q_{n-1} q_{n}\right) E_{n-1} E_{n} E_{n-1}+q_{n} E_{n} E_{n-1}^{2}\right) E_{n}=-\left(1+q_{n-1} q_{n}\right)\left(E_{n-1} E_{n}\right)^{2}+q_{n} E_{n} E_{n-1}^{2} E_{n}=0$

and $E_{n}\left(q_{n-1} E_{n-1}^{2} E_{n}-\left(1+q_{n-1} q_{n}\right) E_{n-1} E_{n} E_{n-1}+q_{n} E_{n} E_{n-1}^{2}\right)=q_{n-1} E_{n} E_{n-1}^{2} E_{n}-\left(1+q_{n-1} q_{n}\right)\left(E_{n} E_{n-1}\right)^{2}=0$.

Since $q_{n}=q_{n-1}$, then we have $\left(E_{n-1} E_{n}\right)^{2}=\left(E_{n} E_{n-1}\right)^{2}$. So $E_{\alpha_{n-1}+\alpha_{n}}^{2}=0$. 
Next we assume that if $i=j+1, E_{j+1, n+1}^{2}=E_{\alpha_{j+1}+\alpha j+2+\ldots+\alpha_{n}}^{2}=0$ set up. By Lemma 4.1, then

$$
\begin{aligned}
E_{j, n+1}^{2} & =\left(a d E_{j}\left(E_{\alpha_{j+1}+\ldots+\alpha_{n}}\right)\right)^{2}=\left(E_{j} E_{\alpha_{j+1}+\ldots+\alpha_{n}}-q_{j+1} E_{\alpha_{j+1}+\ldots+\alpha_{n}} E_{j}\right)^{2} \\
& =\left(E_{j} E_{\alpha_{j+1}+\ldots+\alpha_{n}}\right)^{2}+q_{j+1}^{2}\left(E_{\alpha_{j+1}+\ldots+\alpha_{n}} E_{j}\right)^{2}-q_{j+1} E_{\alpha_{j+1}+\ldots+\alpha_{n}} E_{j}^{2} E_{\alpha_{j+1}+\ldots+\alpha_{n}} \\
& =\left(E_{j} E_{\alpha_{j+1}+\ldots+\alpha_{n}}\right)^{2}+q_{j+1}^{2}\left(E_{\alpha_{j+1}+\ldots+\alpha_{n}} E_{j}\right)^{2}-\left(q_{j+1} q_{j}^{-1}+q_{j+1}^{2}\right)\left(E_{\alpha_{j+1}+\ldots+\alpha_{n}} E_{j}\right)^{2},
\end{aligned}
$$

Also

$$
\begin{aligned}
& E_{\alpha_{j+1}+\ldots+\alpha_{n}}\left(q_{j} E_{j}^{2} E_{j+1, n+1}-\left(1+q_{j} q_{j+1}\right) E_{j} E_{j+1, n+1} E_{j}+q_{j+1} E_{j+1, n+1} E_{j}^{2}\right) \\
= & \left(q_{j} E_{j}^{2} E_{j+1, n+1}-\left(1+q_{j} q_{j+1}\right) E_{j} E_{j+1, n+1} E_{i}+q_{j+1} E_{j+1, n+1} E_{j}^{2}\right) E_{\alpha_{j+1}+\ldots+\alpha_{n}}=0
\end{aligned}
$$

Then we get $\left(E_{\alpha_{j+1}+\ldots+\alpha_{n}} E_{j}\right)^{2}=\left(E_{j} E_{\alpha_{j+1}+\ldots+\alpha_{n}}\right)^{2}$. Since $q_{j}=q_{j+1}=q(j \leq n-1)$, we get

$$
E_{j, n+1}^{2}=\left(\left(1+q_{j+1}^{2}\right)-\left(q_{j+1} q_{j}^{-1}+q_{j+1}^{2}\right)\right)\left(E_{j} E_{\alpha_{j+1}+\ldots+\alpha_{n}}\right)^{2}=0 .
$$

By induction, we have $E_{i, n+1}^{2}=0(1 \leq i \leq n)$. The proof is finished.

Now we prove that $X_{q}\left(A_{n}\right)$ has a triangular decomposition. Let $\tilde{X}_{q}\left(A_{n}\right)$ be an algebra generated by $K_{1}^{ \pm 1}, K_{2}^{ \pm 1}, \ldots, K_{n}^{ \pm 1}, K_{n+1}^{ \pm 1}, E_{1}, \ldots, E_{n}, F_{1}, \ldots, F_{n}$ which satisfies $(\mathrm{R} 1)-(\mathrm{R} 5)$. The left hand side of quantum Serre relation $\left(\mathrm{R}^{\prime}\right)_{(\text {resp. }}\left(\mathrm{R} 8^{\prime}\right)$ ) is denoted by $u_{s}^{+}$(resp. $\left.u_{s}^{-}\right)$. Let $L=\left(\beta_{1}, \beta_{2}, \ldots, \beta_{r}\right)\left(\beta_{i}=\alpha_{j}, 1 \leq j \leq n\right)$ be a finite sequence of simple roots and $E_{L}=E_{\beta_{1}} E_{\beta_{2}} \ldots E_{\beta_{r}} \cdot$ Similar to the proof of [8, 4.12-4.19], the elements $F_{I} K_{1}^{l_{1}} K_{2}^{l_{2}} \ldots K_{n+1}^{l_{n+1}} E_{J}\left(l_{i} \in \mathbb{Z}\right)$ form a basis of $\tilde{X}_{q}\left(A_{n}\right)$, where $I, J$ are finite sequences of simple roots.

Lemma 4. 3. Let $m$ be the multiplication map of $\tilde{X}_{q}\left(A_{n}\right)$, then the image of $m(I \otimes m)$ of $\tilde{X}_{q}^{-}\left(A_{n}\right) \otimes \tilde{X}_{q}^{0}\left(A_{n}\right) \otimes I^{+}$, where $I^{+}$is the two-sided ideal of $\tilde{X}_{q}^{+}\left(A_{n}\right)$ generated by $u_{s}^{+}$and $E_{n}^{2}$, is just a two-sided ideal of $\tilde{X}_{q}\left(A_{n}\right)$.

Proof. Let $V=m(I \otimes m)\left(\tilde{X}_{q}^{-}\left(A_{n}\right) \otimes \tilde{X}_{q}^{0}\left(A_{n}\right) \otimes I^{+}\right)$, then it is spanned by $u u_{s}^{+} E_{n}^{2} E_{L}$, with all sequences $L$, where $u \in \tilde{X}_{q}\left(A_{n}\right)$. It is obvious that $V$ is a left ideal in $\tilde{X}_{q}\left(A_{n}\right)$. We have to show that $V$ is a right ideal in $\tilde{X}_{q}\left(A_{n}\right)$. Let $\Phi$ be the root system corresponding to the same type Lie algebra and $\pi$ be a basis of $\Phi$. Since $E_{n}^{2} F_{i}=F_{i} E_{n}^{2}, E_{n}^{2} F_{i}=F_{i} E_{n}^{2}$, it implies that for all $\gamma \in \pi$, we have $E_{n}^{2} F_{\gamma}=F_{\gamma} E_{n}^{2},\left[E_{n}^{2}, F_{\gamma}\right]=0$.

$$
u_{s}^{+} F_{i}=E_{i}^{2} F_{i} E_{i+1}-\left(q^{-1}+q\right) E_{i} E_{i+1}\left(F_{i} E_{i}+\frac{K_{i+1} K_{i}^{-1}-K_{i} K_{i+1}^{-1}}{q-q^{-1}}\right)+E_{i+1} E_{i}\left(F_{i} E_{i}+\frac{K_{i+1} K_{i}^{-1}-K_{i} K_{i+1}^{-1}}{q-q^{-1}}\right)=F_{i} u_{s}^{+} .
$$

Similarly, we have $u_{s}^{+} F_{i+1}=F_{i+1} u_{s}^{+}, u_{s}^{+} F_{i-1}=F_{i-1} u_{s}^{+}$. It implies that $u_{s}^{+} F_{\gamma}=F_{\gamma} u_{s}^{+},\left[u_{s}^{+}, F_{\gamma}\right]=0$.

Therefore, we have $\left[u_{s}^{+} E_{n}^{2}, F_{\gamma}\right]=u_{s}^{+}\left[E_{n}^{2}, F_{\gamma}\right]+\left[u_{s}^{+}, F_{\gamma}\right] E_{n}^{2}=0 . u\left[u_{s}^{+} E_{n}^{2} E_{L}, F_{\gamma}\right]=u u_{s}^{+} E_{n}^{2}\left[E_{L}, F_{\gamma}\right]$.

Note that $\left[E_{L}, F_{\gamma}\right]$ can be written as the linear combinations of the terms $F_{I^{\prime}} K_{1}^{l_{1}} K_{2}^{l_{2}} \ldots K_{n+1}^{l_{n+1}} E_{J^{\prime}}\left(l_{i} \in \mathbb{Z}\right)$, we have $u u_{s}^{+} E_{n}^{2} E_{L} F_{\gamma}=u F_{\gamma} u_{s}^{+} E_{n}^{2} E_{L}+u u_{s}^{+} E_{n}^{2}\left[E_{L}, F_{\gamma}\right] \in V$. The proof is completed. 
In a similar way, we get

Lemma 4. 4. The image of $I^{-} \otimes \tilde{X}_{q}^{0}\left(A_{n}\right) \otimes \tilde{X}_{q}^{+}\left(A_{n}\right)$ under the map $m(m \otimes I)$ is just the ideal of $\tilde{X}_{q}\left(A_{n}\right)$ generated by $u_{s}^{-}$and $F_{n}^{2}$.

Proposition 4. 5. There exist an isomorphism of $\mathbb{C}$-linear spaces $X_{q}\left(A_{n}\right) \cong X_{q}^{-}\left(A_{n}\right) \otimes X_{q}^{0}\left(A_{n}\right) \otimes X_{q}^{+}\left(A_{n}\right)$.

Proof. The proof is similar to [8, Theorem 4.21].

Let $\gamma_{1}<\gamma_{2}<\ldots<\gamma_{N}$, where $N$ the number of positive roots and $\gamma_{i}$ runs over all positive roots.

Lemma 4. 6. $\mathbf{B}^{+}=\left\{E_{\gamma_{1}}^{n_{1}} E_{\gamma_{2}}^{n_{2}} \ldots E_{\gamma_{N}}^{n_{N}} \mid n_{i} \in \mathbb{Z}_{2}\right.$ if $E_{\gamma_{i}}=E_{j, k}(k=n+1), n_{i} \in \mathbb{Z}_{\geq 0}$ if $\left.E_{\gamma_{i}}=E_{j, k}(k \leq n)\right\}$ form a basis of $X_{q}^{+}\left(A_{n}\right)$.

Proof. We show that any element of $X_{q}^{+}\left(A_{n}\right)$ is a linear combinations of $E_{\gamma_{1}}^{n_{1}} E_{\gamma_{2}}^{n_{2}} \ldots E_{\gamma_{N}}^{n_{N}}$ and $E_{\gamma_{1}}^{n_{1}} E_{\gamma_{2}}^{n_{2}} \ldots E_{\gamma_{N}}^{n_{N}}$ are linearly independent with all $E_{\gamma_{1}}^{n_{1}} E_{\gamma_{2}}^{n_{2}} \ldots E_{\gamma_{N}}^{n_{N}} \neq 0$.

(1) It is noted that $E_{1}, \ldots, E_{N}$ generate $X_{q}^{+}\left(A_{n}\right)$. It is suffices to show that $E_{\gamma_{i_{1}}} E_{\gamma_{i_{2}}} \ldots E_{\gamma_{i_{k}}}$ is a linear combination of $E_{\gamma_{1}}^{n_{1}} E_{\gamma_{2}}^{n_{2}} \ldots E_{\gamma_{N}}^{n_{N}}$ with $n_{1}+n_{2}+\ldots+n_{N} \leq k$. Using induction on $k$, given an integer $i$, if $k=1$, then the case is clear. Suppose the assertion holds for $k$. If $i_{1}=1$, by the induction on $k$ to the element $E_{\gamma_{i_{2}}} \ldots E_{\gamma_{i_{k+1}}}$, then we get the result. If $i_{1}>1$, by the induction it is easy to see that $E_{\gamma_{i_{1}}} E_{\gamma_{i_{2}}} \ldots E_{\gamma_{i_{k+1}}}$ is a linear combination of $E_{\gamma_{i_{1}}} E_{\gamma_{j}}^{n_{j}} \ldots E_{\gamma_{N}}^{n_{N}}$ with $n_{j}+n_{2}+\ldots+n_{N} \leq k$.

a) If $i_{1} \leq j$, then it is straightforward to see.

b) If $i_{1}>j, E_{\gamma_{i_{1}}} E_{\gamma_{j}}^{n_{j}} \ldots E_{\gamma_{N}}^{n_{N}}=E_{\gamma_{i_{1}}} E_{\gamma_{j}} E_{\gamma_{j}}^{n_{j}-1} \ldots E_{\gamma_{N}}^{n_{N}}$, by lemma 3.4, for some non-zero coefficients $\lambda, \mu$, $E_{\gamma_{i_{1}}} E_{\gamma_{j}}-\lambda E_{\gamma_{j}} E_{\gamma_{i_{1}}}=\left\{\begin{array}{l}0, \\ \mu E_{\gamma_{i_{1}}+\gamma_{j}}, \\ \mu E_{\gamma_{i_{1}}+\gamma_{j}-\gamma^{\prime}} E_{\gamma^{\prime}}, \quad \text { where } \gamma_{i_{1}}=\alpha^{\prime}+\gamma^{\prime}, \gamma_{j}=\gamma^{\prime}+\beta^{\prime},\end{array}\right.$

Then

$$
E_{\gamma_{i_{1}}} E_{\gamma_{j}} E_{\gamma_{j}}^{n_{j}-1} E_{\gamma_{j+1}}^{n_{j+1}} \ldots E_{\gamma_{N}}^{n_{N}}=\left\{\begin{array}{l}
\lambda E_{\gamma_{j}} E_{\gamma_{i_{1}}} E_{\gamma_{j}}^{n_{j}-1} E_{\gamma_{j+1}}^{n_{j+1}} \ldots E_{\gamma_{N}}^{n_{N}}, \\
\lambda E_{\gamma_{j}} E_{\gamma_{i_{1}}} E_{\gamma_{j}}^{n_{j}-1} E_{\gamma_{j+1}}^{n_{j+1}} \ldots E_{\gamma_{N}}^{n_{N}}+\mu E_{\gamma_{i_{1}}+\gamma_{j}} \lambda E_{\gamma_{j}} E_{\gamma_{i_{1}}} E_{\gamma_{j}}^{n_{j}-1} E_{\gamma_{j+1}}^{n_{j+1}} \ldots E_{\gamma_{N}}^{n_{N}}, \\
\lambda E_{\gamma_{j}} E_{\gamma_{i_{1}}} E_{\gamma_{j}}^{n_{j}-1} E_{\gamma_{j+1}}^{n_{j+1}} \ldots E_{\gamma_{N}}^{n_{N}}+\mu E_{\gamma_{i_{1}}+\gamma_{j}-\gamma^{\prime}} E_{\gamma^{\prime}} \lambda E_{\gamma_{j}} E_{\gamma_{i_{1}}} E_{\gamma_{j}}^{n_{j}-1} E_{\gamma_{j+1}}^{n_{j+1}} \ldots E_{\gamma_{N}}^{n_{N}} .
\end{array}\right.
$$

Similar to the proof in [9, Proposition 5.3] and [7], we get that $E_{\gamma_{i_{1}}} E_{\gamma_{i_{2}}} \ldots E_{\gamma_{i_{k}}}$ is a linear combination of $E_{\gamma_{1}}^{n_{1}} E_{\gamma_{2}}^{n_{2}} \ldots E_{\gamma_{N}}^{n_{N}}$ with $n_{1}+n_{2}+\ldots+n_{N} \leq k$

(2) We now show that all $E_{\gamma_{1}}^{n_{1}} E_{\gamma_{2}}^{n_{2}} \ldots E_{\gamma_{N}}^{n_{N}} \neq 0$ and $E_{\gamma_{1}}^{n_{1}} E_{\gamma_{2}}^{n_{2}} \ldots E_{\gamma_{N}}^{n_{N}}$ are linearly independent by comultiplication $\Delta$. Firstly, one can prove that

$$
\left[E_{i, j+1}, F_{i, j+1}\right]=\frac{(-q)^{j-i}\left(K_{i}^{-1} K_{j+1}-K_{i} K_{j+1}^{-1}\right)}{q-q^{-1}}
$$


by induction on $j-i$, we get that $E_{i, n+1}^{2}=0(i \leq n)$ and $E_{i, j+1}^{l} \neq 0(1 \leq i \leq j<n)$ for all $l$.

The root lattice $Q$-gradation of $X_{q}^{+}\left(A_{n}\right)$ implies that $E_{\gamma_{j}}{ }^{\prime}$ are independent. Note that

$\Delta: X_{q}^{+}\left(A_{n}\right) \rightarrow X_{q}^{\geq 0}\left(A_{n}\right) \otimes X_{q}^{+}\left(A_{n}\right)$ is a graded algebra homomorphism and $\Delta\left(E_{\beta}\right)$ has a component of bidegree $\left(\alpha_{i}, \beta-\alpha_{i}\right)$ if and only if $\beta=\alpha_{i}+\left(\beta-\alpha_{i}\right)$. It follows that for the positive root $\beta=\varepsilon_{i}-\varepsilon_{j}(i<j<n+1)$ the component of bidegree $\left(l \alpha_{i}, l\left(\beta-\alpha_{i}\right)\right)$ of $\Delta\left(E_{\beta}\right)^{l}$ is proportional to $E_{i}^{l} K_{\beta-\alpha_{i}}^{l} \otimes E_{\beta-\alpha_{i}}^{l}$ for any $l$, and for the positive $\operatorname{root} \beta=\varepsilon_{i}-\varepsilon_{n+1}(i<n+1)$ the component of bidegree $\left(\alpha_{i}, \beta-\alpha_{i}\right)$ of $\Delta\left(E_{\beta}\right)$ is proportional to $E_{i} K_{\beta-\alpha_{i}} \otimes E_{\beta-\alpha_{i}}$. The rest is similar to the proof in [7] and [9, Proposition 5.3] . By Lemma 3.5, we get that $E_{\gamma_{1}}^{n_{1}} E_{\gamma_{2}}^{n_{2}} \ldots E_{\gamma_{N}}^{n_{N}}$ are linearly independent. The proof is finished.

Similarly, we have

Lemma 4. 7 .

$$
\mathbf{B}^{-}=\left\{F_{\gamma_{1}}^{m_{1}} F_{\gamma_{2}}^{m_{2}} \ldots F_{\gamma_{N}}^{m_{N}} \mid m_{i} \in \mathbb{Z}_{2} \text { if } F_{\gamma_{i}}=F_{j, k}(k=n+1), m_{i} \in \mathbb{Z}_{\geq 0} \text { if } F_{\gamma_{i}}=F_{j, k}(k \leq n)\right\}
$$

form a basis of $X_{q}^{-}\left(A_{n}\right)$.

Let $E^{s}=E_{\gamma_{1}}^{n_{1}} E_{\gamma_{2}}^{n_{2}} \ldots E_{\gamma_{N}}^{n_{N}}, F^{t}=F_{\gamma_{1}}^{m_{1}} F_{\gamma_{2}}^{m_{2}} \ldots F_{\gamma_{N}}^{m_{N}}, \quad$ where $\quad s=\left(n_{1}, n_{2}, \ldots, n_{N}\right), t=\left(m_{1}, m_{2}, \ldots, m_{N}\right) . \quad$ If $E_{\gamma_{i}}=E_{j, n+1} \quad$ (resp. $\quad F_{\gamma_{i}}=F_{j, n+1}$ ), then $n_{i} \in \mathbb{Z}_{2} \quad$ (resp. $\quad m_{i} \in \mathbb{Z}_{2}$ ); if $\quad E_{\gamma_{i}}=E_{j, k}(k \leq n)$ (resp. $F_{\gamma_{i}}=F_{j, k}(k \leq n)$ ), then $n_{i} \in \mathbb{Z}_{\geq 0}$ (resp. $m_{i} \in \mathbb{Z}_{\geq 0}$ ). The elements in $\mathbf{B}^{0}=\left\{K_{1}^{l_{1}} K_{2}^{l_{2}} \ldots K_{n+1}^{l_{n+1}} \mid l_{i} \in \mathbb{Z}\right\}$ is denoted by $K^{l}$, we have

Theorem 4. 8. $\mathbf{B}=\left\{F^{t} K^{l} E^{s} \mid E^{s} \in \mathbf{B}^{+}, F^{t} \in \mathbf{B}^{-}, K^{l} \in \mathbf{B}^{0}\right\}$ form a basis of $X_{q}\left(A_{n}\right)$.

\section{Acknowledgment}

This work is supported by the National Natural Science Foundation of China (Grant No. 11471186, 11671024) and the Beijing Natural Science Foundation (Grant No. 1162002).

\section{References}

[1] Ge, M., Liu, G., \& Xue, K. (1991). New solutions of Yang-Baxter equations: Birman-Wenzl algebra and quantum group structures. J. Phys. A: Math. Gen., 24, 2679-2690.

[2] Jing, N., Ge, M., \& Wu, Y. (1991). A new quantum group associated with a 'nonstandard' braid group represention. Lett. Math. Phys., 21,193-203.

[3] Aghamohammadi, A., Karimipour, V., \& Rouhani, S. (1993). The multiparametric non-standard deformation of $A_{n-1}$.J. Phys. A: Math. Gen., 26, L75-L82.

[4] Aghamohammadit, A., Karimipourt, V., \& Nezami, A. R. (1994). Non-standard deformation of $B_{n}$ series. J. Phys. A: Math. Gen., 27, 1609-1616.

[5] Cheng, C., \& Yang, S. (2017). Weak Hopf algebras corresponding to non-standard quantum groups. Bull. Korean Math. Soc., 54(2), 463-484.

[6] Kassel, C. (1995). Quantum Groups. GTM 155. Springer-verlag. 1-50.

[7] Rosso, M. (1989). An analogue of P.B.W. theorem and the universal R-matrix for $U_{h} s l(N+1)$. Comm. Math. Phys., 124(2), 307-318. 
[8] Jantzen, J. C. (1995). Lectures on quantum groups. Grad. Stud. Math., 6, Amer. Math. Soc.

[9] Ai, C., \& Yang, S. (2016). Two-parameter quantum superalgebras and PBW theorem. Algebra Colloq., 23(2), 303-324.

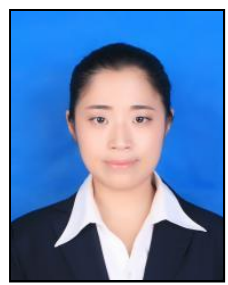

Cheng Cheng received a master degree in 2014 from Qufu Normal University, Shandong, China. She is now pursuing the PhD degree from 2014 in College of Applied Sciences, Beijing University of Technology, Beijing, China. Her research interests include algebra and its applications, representation theory of algebras, structures and representations of quantum groups, and (weak) Hopf algebras.

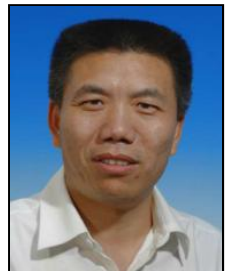

Shilin Yang works as a professor, $\mathrm{PhD}$ advisor in college of applied sciences, Beijing University of Technology. He received a PhD degree from the Department of mathematics, Beijing Normal University, Beijing, China. His research interests include algebra and its applications, representation theory of algebras, structures and representations of quantum groups, Ringel-Hall algebras and (weak) Hopf algebras. 